\title{
La recuperación de los elementos de piedra en el patrimonio arquitectónico monumental: el caso del claustro del convento de la Asunción de Calatrava de Almagro (Ciudad Real)
}

\section{Recovery of stone elements in monumental architectural heritage: the case of Asunción de Calatravas' cloister in Almagro (Ciudad Real)}

\author{
L. Maldonado-Ramos ${ }^{(*)}$, M. M. Barbero-Barrera ${ }^{(*)}$, D. Rivera-Gámez ${ }^{(*)}$
}

\section{RESUMEN}

El Claustro del Convento de la Asunción de Calatravas es uno de los ejemplos de arquitectura renacentista de mayor interés situados en la provincia de Ciudad Real.

El presente artículo expone y resume el proceso de intervención llevado a cabo en los elementos pétreos del claustro, fundamentado en una investigación teórica sobre el tipo de material existente en la zona de estudio y en la edificación así como en las técnicas de intervención más adecuadas. Una vez seleccionadas éstas, su adecuación y compatibilidad se comprobó a partir de ensayos in situ desarrollados en áreas específicas de la intervención, extendiéndose posteriormente al resto de la actuación.

El artículo pretende divulgar la metodología empleada así como la actuación llevada a cabo en dicha edificación y crear un foro de debate sobre las técnicas de actuación restauradora en casos similares de patrimonio arquitectónico renacentista.

Palabras clave: Piedra; restauración; Renacimiento; limpieza; consolidación; reintegración.

\section{ABSTRACT}

The cloister of Asunción de Calatravas' Convent is one of the most important examples of Renaissance in Ciudad Real province.

This paper compiles the explanation and summary of the intervention process in the stone elements of the cloister, based on a theoretical research about the type of stones in the area of analysis and the building as well as the most suitable techniques to restore them. Once the latter where selected, their adequation and compatibility was checked by on site tests.

The paper has as main aim to divulge the methodology and the intervention, as well as to create a forum of debate about the restoration techniques for similar cases of Renaissance Architectural Heritage.

Keywords: Stone; restoration; Renaissance; cleaning; consolidation; reintegration.

(*) Escuela Técnica Superior de Arquitectura-Universidad Politécnica de Madrid Persona de contacto/Corresponding author: mar.barbero@upm.es (M. M. Barbero-Barrera)

Cómo citar este artículo/Citation: Maldonado-Ramos, L., Barbero-Barrera, M.M., Rivera-Gámez, D. (2014). La recuperación de los elementos de piedra en el patrimonio arquitectónico monumental: el caso del claustro del convento de la Asunción de Calatrava de Almagro (Ciudad Real). Informes de la Construcción, 66(536): e049, doi: http://dx.doi.org/10.3989/ic.13.146.

Licencia/License: Salvo indicación contraria, todos los contenidos de la edición electrónica de Informes de la Construcción se distribuyen bajo una licencia de uso y distribución Creative Commons Reconocimiento no Comercial 3.o. España (cc-by-nc). 


\section{INTRODUCCIÓN}

El Convento de la Asunción de Calatrava (Figura 1 y 2) forma parte del conjunto histórico de la ciudad de Almagro y es uno de los ejemplos renacentistas más importantes de la provincia de Ciudad Real, encontrándose ubicado a la entrada de la ciudad, en el margen del núcleo histórico.

Datado de mediados del siglo XVI, sus trazas responden a las exigencias estilísticas y funcionales del momento con una tipología pura y refinada en cuanto a acabados se refiere. En él se combinan elementos tardogóticos, mudéjares, platerescos y renacentistas con influencias del Quattrocento y el Cinquecento italianos marcadas por ejemplo en las portadas clásicas y en la esbeltez y ligereza de las arquerías del claustro.

De planta casi cuadrada, el claustro fue construido a finales del siglo XVI y es sin duda el elemento histórico-artístico más importante del convento. Su trazado se debe casi con toda certeza a Enrique Egas el Mozo, y responde al modelo de San Juan de los Reyes de Toledo, que lógicamente resultaba muy familiar al arquitecto por haber sido proyectado por su propio padre.

El claustro presenta ocho intercolumnios en el lado de la sala capitular y del refectorio, y siete tramos en las pandas opuestas, aunque el efecto de regularidad se obtiene fácilmente mediante la armónica disposición de los arcos. El claustro consta de dos galerías superpuestas con órdenes clásicos, toscano en la planta superior y jónico en la inferior, en una clara referencia a los modelos establecidos por los tratados arquitectónicos que empezaban a difundirse por España (Medias del romano, de Diego de Sagredo, el primer tratado español de arquitectura, datado en 1526 y fuertemente influido por Vitruvio, puede considerarse quizá una buena fuente para la resolución de algunos de los elementos arquitectónicos e iconográficos, como los motivos de candelieri o la propia configuración de los capiteles).

Del mismo modo, el programa iconográfico, los espejos circulares convexos, las molduras de los arcos y la balaustrada responden al modelo italiano, mientras que la simbología plateresca queda patente en relieves y acabados de portadas y ventanas (Figura 3).

Sobre el conjunto se han llevado a cabo continuas actuaciones de mantenimiento y conservación (1) (2) (3). Las primeras de una larga serie, recogidas en el Archivo Judicial de Toledo, datan de 1609 y 1682. En 1854 el edificio fue situado bajo la

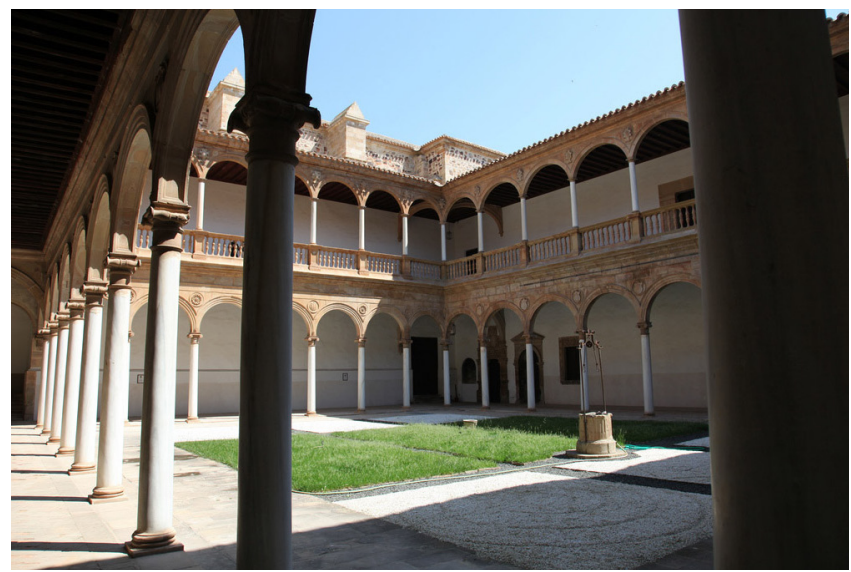

Figura 2. Vista general del claustro.

tutela de la Comisión Provincial de Monumentos, emprendiéndose obras de conservación que serían paralizadas en 1860 al desaparecer la propia Comisión. En 1893 se redacta un proyecto de restauración por parte de la Real Academia de Bellas Artes de San Fernando, debido al avanzado estado de deterioro que presentaba el convento, basándose en este caso en un informe emitido por el Arquitecto municipal del momento, Sebastián Rebollos. Aquel, sin embargo, no llegará a ponerse en obra y habrá que esperar hasta 1903, cuando una comunidad de dominicos se traslada al edificio, para que se lleven a cabo distintas obras de intervención que darían lugar a diversas modificaciones en el trazado original del conjunto.

La Guerra Civil dejó un saldo de numerosos daños infligidos al conjunto, pero la restauración consecuente del convento no se llevará a la práctica hasta entrados los años 70. En 1978 Santiago Camacho (4) dirige la restauración de las pandas oeste y sur del Claustro, consistente en la reparación de «los forjados y artesonado del ala norte, además de pavimentando con baldosas de barro cocido similares a las que aún quedaban por el suelo y arriostrando la arquería del claustro» (véase una breve memoria de estas transformaciones en Maldonado et al. 2002: 46). A éstas les siguen en 1979 las obras de restauración integral. En 1980-1982, Miguel Fisac restaura la torre de la iglesia y construye una estructura nueva de hormigón armado en el interior de la misma, con objeto de atar la obra de fábrica antigua y poder mantener su uso. En 1988 Carmen Sánchez redacta un nuevo proyecto de restauración al tiempo que Francisco Racionero acomete la rehabilitación del colegio dominico para alojar una hospedería. La conclusión de las obras, en 1991, coincide con el encargo del proyec-
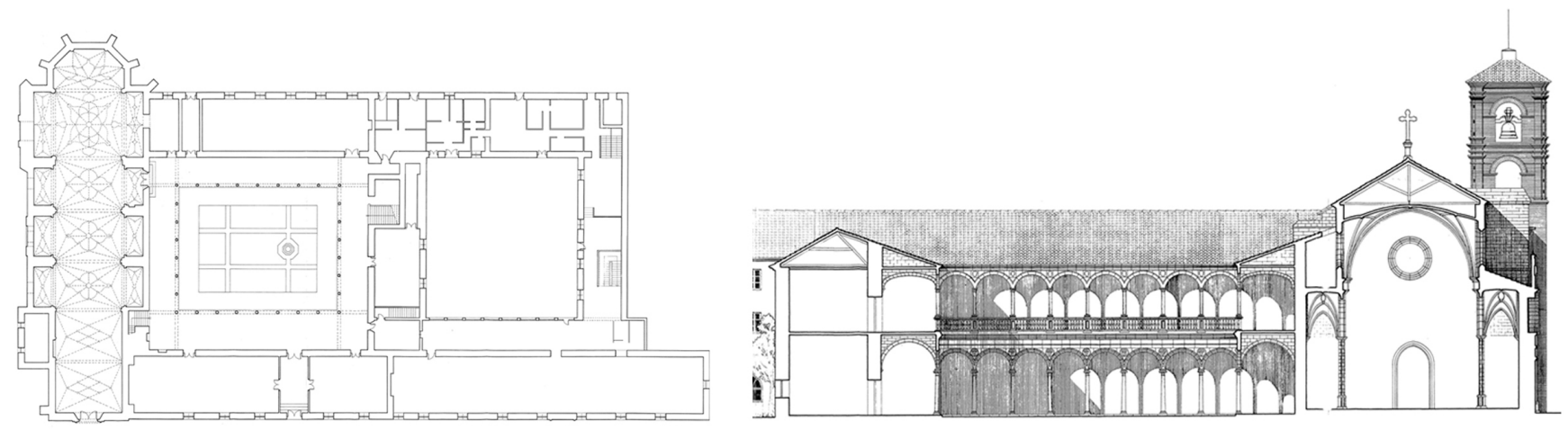

Figura 1. Planta baja y alzado-sección longitudinal. 


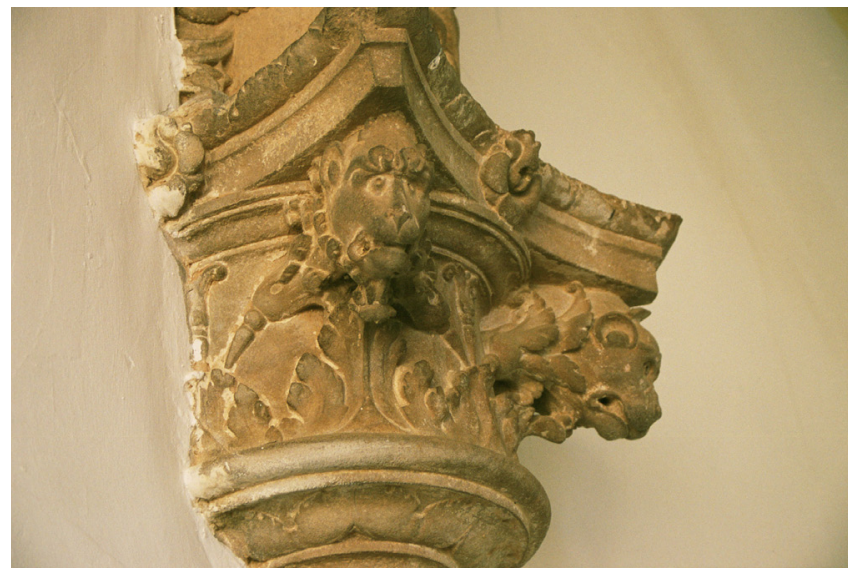

Figura 3. Ejemplo de capitel decorado.

to de restauración de Iglesia y Claustro al arquitecto Francisco Jurado, quien aborda la consolidación de los contrafuertes y la eliminación de las humedades, contemplando también la reposición de cornisas y de la sillería perdida. En 2002 se desarrolla el Plan Director de Restauración del Convento por parte del equipo dirigido por el arquitecto Luis Maldonado, plan que tiene como objetivos esenciales establecer las fases constructivas y económicas adecuadas para la restauración integral del conjunto y su necesaria puesta en valor, teniendo en cuenta las necesidades del cliente y las correspondientes disposiciones presupuestarias (5) (6) (7). La restauración descrita en el presente artículo, realizada en colaboración con la empresa Artemon dirigida por Vicente Aguilar, corresponde a una sola de las fases contempladas, la relativa a la restauración y consolidación de los elementos pétreos ubicados en el claustro del convento.

\subsection{Estado de conservación de la piedra}

El material objeto de la intervención es, en su mayor parte, arenisca, una roca sedimentaria local de tonos pardos. Almagro, ubicado en la submeseta sur, cuenta con un clima caracterizado por fuertes oscilaciones térmicas estacionales y diarias que, provocan elevadas tensiones sobre los materiales constructivos, especialmente sobre los expuestos a la radiación solar. El choque térmico es una de las causas de daños más frecuentes en Castilla donde la reiteración de dichos cambios bruscos de temperaturas genera tensiones entre las capas superficiales y las interiores de las piedras, fomentado por la anisotropía característica de los carbonatos, siendo éstas una de las causas fundamentales del desprendimiento de lajas superficiales (7) (8).

Estas tensiones se acentúan con el elevado número de días de heladas que, en esta región, alcanzan prácticamente 50 días/ año (9) (10) así como la fuerte insolación que sufre la zona (9) siendo dichas circunstancias especialmente importantes en el caso de materiales porosos (8). La piedra objeto de intervención presentaba un avanzado estado de degradación por diversos factores, entre ellos, los acusados ciclos de hielo y deshielo y la fuerte irradiación solar, siendo éstos especialmente relevantes en los elementos más expuestos como cornisas e impostas. Tal y como se puede apreciar en la Figura 4, en ellas se apreciaban importantes pérdidas volumétricas y roturas parciales. La escasa dureza del material pétreo favorecía, además, los procesos patológicos observándose avanzados procesos de disgregación y de arenización.

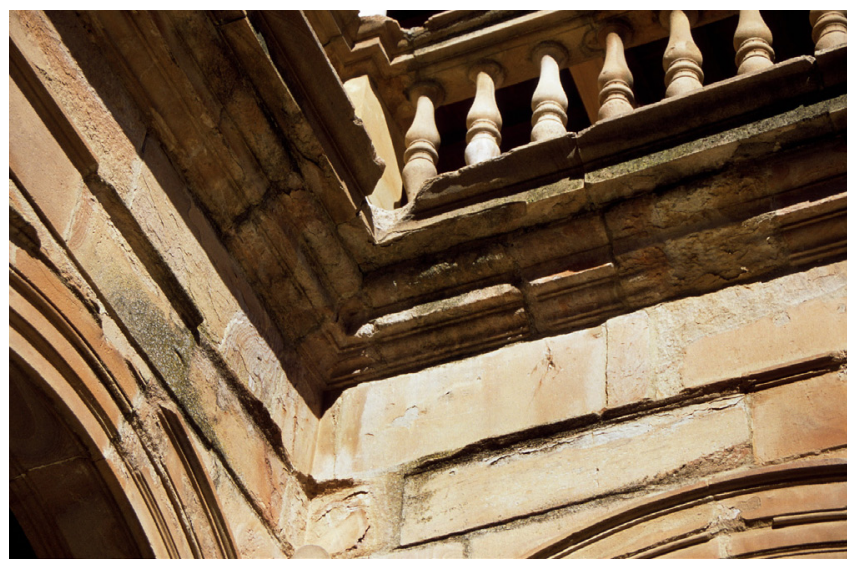

Figura 4. Detalle de imposta en la que se aprecia la pérdida de material pétreo en la esquina así como distintos procesos patológicos en la superficie de la piedra provocados por la filtración y escorrentía de agua.

Por otra parte, la presencia de contaminantes atmosféricos generados por el tráfico rodado y la cercanía de una zona industrial había derivado en la presencia de costras negras (7) (11) (12) en las zonas más protegidas de los elementos escultóricos.

Junto con los condicionantes climáticos, las actuaciones efectuadas con anterioridad habían acelerado el proceso de degradación mediante la incorporación de materiales incompatibles con los originales. En concreto, la introducción de morteros de cemento, más rígidos y resistentes que los originales con base de cal, y con un coeficiente de dilatación térmica sustancialmente diferente del de los elementos pétreos, había generado la aparición de tensiones concentradas en estos últimos. Asimismo, los cambios de volumen correspondientes a los diversos estados de cristalización de las sales solubles con frecuencia presentes en los cementos generaban presiones sobre las paredes de los capilares de los materiales pétreos que, a medio-largo plazo, derivan en su rotura.

En el caso de la fábrica de piedra del claustro, este tipo de mortero se encontraba no sólo en juntas sino que también se había empleado en la reintegración de algunos elementos como cornisas de alero, dovelas de arcos y uniones de capiteles. En algunos casos, las criptoeflorescencias habían generado procesos avanzados de arenización.

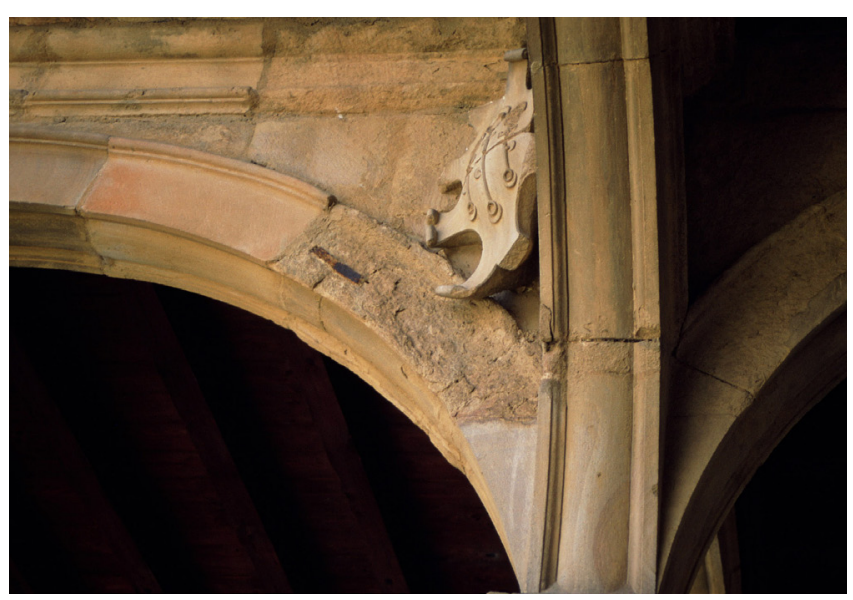

Figura 5. Ejemplo de grapa metálica en la unión entre dovelas y sobrecementación en la arenisca. 
Por otra parte, el empleo de grapas metálicas (Figura 5) para la unión mecánica de las piedras había provocado tinciones y la rotura de piezas debido a las tensiones generadas por el incremento de volumen del proceso de oxidación y corrosión (13) (14). Finalmente, la piedra presentaba procesos de biodeterioro (algas, musgos y líquenes) causados por la falta de mantenimiento y la humedad (Figura 6) así como procesos de disgregación granular o arenización (7).

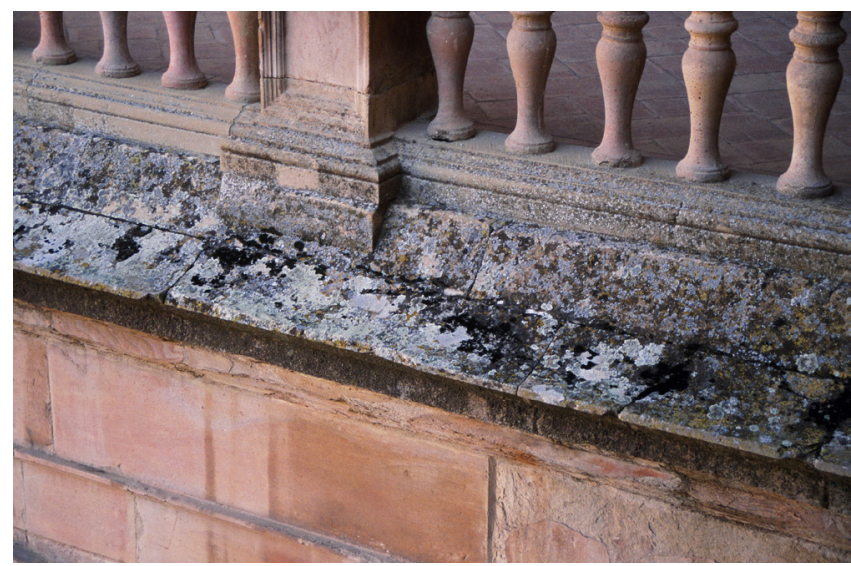

Figura 6. Detalle de microorganismos vegetales en cornisa intermedia.

A partir del análisis del caso de estudio así como también como extracto de la bibliografía consultada (7) (15), proponemos dos cuadros que recogen y analizan la degradación en los materiales pétreos: Cuadro 1 - Degradación por causas medioambientales (origen abiótico) y Cuadro 2 - Degradación por causas biológicas (origen biótico), que se resumen en la Tabla 1, recogida al final del presente apartado.

\section{Cuadro no ${ }^{\circ}$ de degradación por causas medioambientales (Origen Abiótico)}

\section{A. Alteraciones de la superficie}

Las alteraciones en la superficie de los materiales pétreos son capas o películas que se desarrollan en la superficie exterior de los mismos y que se encuentra expuesta a los agentes medioambientales.

A.1. Tinciones: Son los cambios de color producidos en el aspecto exterior del material.

A.2. PÁtinas: Modificaciones superficiales de poco espesor. No implican necesariamente procesos de degradación.

A.3. Costras: Alteración de la superficie exterior del material con mayor espesor que las pátinas y que generan procesos de deterioro. Se generan por microorganismos o por depósito de otros materiales.

A.4. EFlorescencias: Aparición de sales en la superficie, producidas por efecto de la evaporación, en los capilares exteriores de disoluciones que contienen dichas sales.

\section{B. Pérdida de material}

Son daños cuyo efecto es la disminución del espesor del material, alterando la forma original de la piedra.

B.1. ERosión: Daños generados por procesos de abrasión, alveolización u otros
B.2. Decementación: Cuando el proceso patológico afecta al compuesto que da cohesión al material (aglomerantes o conglomerantes) generando la pérdida de dicho material por falta de unión entre moléculas o sistemas, generalmente por alteración química de sus minerales.

B.3. ArEnización. Se produce cuando se da un proceso de desprendimiento de la masa del material en granos (generalmente mayor de $1 \mathrm{~mm}$ de diámetro) Puede estar asociado a los procesos antes descritos.

B.4. Disgregación: Cuando el proceso patológico produce una separación de los diferentes componentes del material.

B.5. Descomposición: Es la denominación genérica de los daños anteriores que supone la alteración del material pétreo y la pérdida del mismo. Puede ser por causa físicas (generalmente mecánicas), químicas o biológicas. También se denomina meteorización.

\section{Roturas y disyunciones}

Son la consecuencia de procesos patológicos en el material pétreo que producen separación de partes de la piedra. Estos procesos pueden tener su origen en acciones mecánicas (crioclásticas, termoclásticas o hidroclásticas)

C.1. Fisuras: Abertura lineal no controlada que se produce por causa de un proceso patológico. Su ancho es menor de $1 \mathrm{~mm}$. y puede no afectar a todo el elemento constructivo.

C.2. Grietas: Al igual que la fisura es una hendidura no controlada pero su ancho suele ser mayor de $1 \mathrm{~mm}$. Y afecta a todo el elemento constructivo.

C.3. Fracturas: Cuando, además de la grieta se producen desplazamientos de las partes resultantes. Este desplazamiento puede ser de plomo, de nivel o ambos.

C.4. Picaduras: Desprendimiento selectivo de parte del material en zonas localizadas.

C.5. Desprendimiento: Pérdida de una parte del material que se inicia por la cara exterior más expuesta. Según su efecto recibe varios nombres, exfoliación, escamación, ampollas, desconchado, etc.

\section{Cuadro no 2 degradación por causas biológicas}

\section{Alteraciones superficiales de origen biótico}

Corresponde a todos los daños cuyo origen son los seres vivos, y se clasifica en función de la naturaleza de los mismos.

D.1. MICROORGANISMOS: Son seres vivos que tienen una organización biológica elemental, como las bacterias, los hongos, levaduras, etc. Estos microorganismos pueden ser patógenos, cuando producen alteraciones perjudiciales, pero también pueden ser agentes reparadores que se aplican para rehacer los productos naturales.

D.2. PLANTAS: Son seres vivos fotosintéticos sin capacidad locomotora. Crecen en las piedras, en las juntas de su aparejo o entre elementos constructivos. Generalmente surgen cuando ya se ha iniciado un proceso patológico que produce las condiciones para que éstas puedan germinar (depósitos, arenizaciones, etc.)

D.3. ANIMALEs: Son seres vivos con capacidad locomotora y metabolismo aerobio (consume oxígeno). Los daños que pueden producir en las piedras son raros y escasos. 
Tabla 1. Cuadro resumen de las causas de la degradación de materiales pétreos.

\begin{tabular}{|c|c|}
\hline \multicolumn{2}{|c|}{ Degradación de Materiales Pétreos } \\
\hline $\begin{array}{l}\text { Origen Abiótico: } \\
\text { Degradación provocada por causas medioambientales }\end{array}$ & $\begin{array}{l}\text { Origen Biótico: } \\
\text { Degradación provocada por causas biológicas }\end{array}$ \\
\hline $\begin{array}{l}\text { 1. Alteraciones superficiales } \\
\text { 1.1. Eflorescencias } \\
\text { 1.2. Tinciones } \\
\text { 1.3. Pátinas } \\
\text { 1.4. Costras }\end{array}$ & $\begin{array}{l}\text { 1. Alteraciones superficiales de origen biótico } \\
\text { 1.1. Microorganismos } \\
\text { 1.2. Plantas } \\
\text { 1.3. Animales }\end{array}$ \\
\hline $\begin{array}{l}\text { 2. Pérdida del material } \\
\text { 2.1. Erosión } \\
\text { 2.2. Decementación } \\
\text { 2.3. Arenización } \\
\text { 2.4. Disgregación } \\
\text { 2.5. Descomposición }\end{array}$ & $\begin{array}{l}\text { 2. Alteraciones superficiales de origen antrópico } \\
\text { 2.1. Pintadas } \\
\text { 2.2. Grafitis }\end{array}$ \\
\hline $\begin{array}{l}\text { 3. Roturas y disyunciones } \\
\text { 3.1. Fisuras } \\
\text { 3.2. Grietas } \\
\text { 3.3. Fracturas } \\
\text { 3.4. Picaduras } \\
\text { 3.5. Desprendimientos }\end{array}$ & $\begin{array}{l}\text { 3. Demoliciones } \\
\text { 4. Degradación generalizada }\end{array}$ \\
\hline
\end{tabular}

\section{E. Alteraciones superficiales de origen antrópico}

E.1. PinTADAS: Son agresiones de carácter marginal que se realizan sobre edificios para manifestar una opinión y consiste en aplicar pinturas sobre las fábricas.

E.2. GRAFITIS: Como en las pintadas son agresiones marginales pero que se producen por una falta de sensibilidad social al patrimonio construido, o por un desprecio del mismo generado por la incultura.

\section{F. Demoliciones}

Cuando no existe una normativa que proteja el patrimonio o existe un desconocimiento por falta de estudio o puesta en valor, nos podemos encontrar con casos de demolición total o parcial de fábrica de piedra e incluso de edificios completos.

\section{G. Degradación generalizada}

La falta de conservación y mantenimiento, el uso inadecuado o la realización de obras que alteran el estado del edificio pueden ser origen de deterioro con carácter general en la fábrica de piedra. Las consideramos de origen biológico por ser la omisión de la conservación y mantenimiento por parte de los usuarios o de los propietarios de los inmuebles, lo que llega a generar esta degradación.

\section{PROCESO DE INTERVENCIÓN}

Las zonas de actuación estaban compuestas por elementos de piedra sedimentaria. Tal y como contemplaba el Plan Director de Restauración, la actuación supuso la limpieza de los elementos así como también su preconsolidación y tratamiento. También se contemplaron la reintegración parcial y la eliminación total de los rejuntados elaborados con morteros de cemento. Estas actuaciones, en aras de la compatibilidad con los soportes antiguos serían realizadas tras su comprobación sobre el material existente, considerando además la puesta en obra mediante los procedimientos adecuados (16).

El proceso de intervención constó de varias fases: limpieza, consolidación, reintegración de piezas, tratamiento de juntas y recuperación cromática.

\subsection{Saneado y limpieza}

El primer paso consistió en la eliminación de los microorganismos vegetales y de los morteros de cemento (Figura 7). Para asegurar la efectividad de los tratamientos biocidas (15) y evitar daños sobre las piedras, aquéllos fueron aplicados sobre las piedras secas en dilución de agua 1/50-1/100. Junto con esta limpieza se realizó un posterior saneado de la superficie eliminando el material desprendido mediante cepillado y dejando a la vista la parte del material sano para su posterior consolidación (15). Los morteros de mayor dureza aplicados en restauraciones anteriores, fueron eliminados con un sistema mecánico por rotación previa, hasta alcanzar el material sano. Para evitar daños en los elementos originales, previamente se comprobó el estado de la zona de contacto entre dichos morteros y la piedra, valorando la posibilidad de actuar sobre la misma.

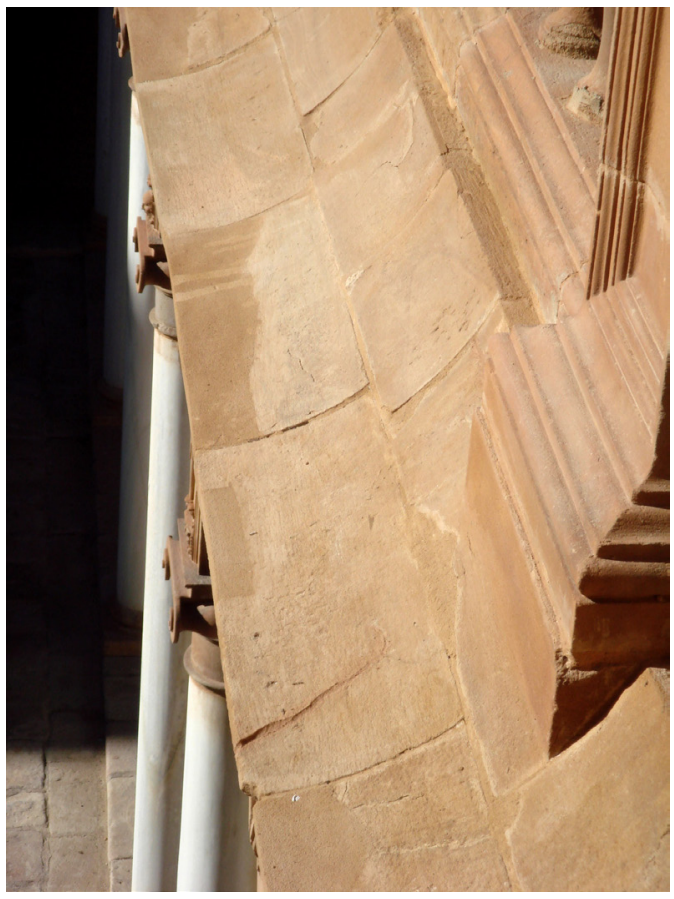

Figura 7. Detalle de la imposta después del proceso de limpieza y tratamiento biocida. 
El sistema de limpieza general elegido fue determinado por los estudios previos efectuados sobre las piedras y consistió en agua a baja presión. Siendo el agua un magnífico diluyente de la mayoría de las sales dañinas. Este método puede ser desaconsejado en determinados casos como, por ejemplo, en piedras altamente absorbentes. En el caso de las zonas de rejuntado, se prestó especial atención al hecho de que no penetrase al interior de una cantidad de agua no deseada. Respecto a dicho sistema, uno de los puntos fundamentales fue la determinación de la presión ejercida, que fue cuidadosamente seleccionada para que la pátina característica de la piedra se mantuviera, al tiempo que se evitaran los daños sobre ésta ejercidos tanto por la presión como por la penetración de humedad. A pesar de que la presión ideal de uso suele ser de 1 a 3 atmósferas, ésta puede alcanzar las 4-5 atmósferas en el caso de piedras de cierta dureza. En este caso, la presión de impacto ideal adoptada fue de 3,0-3,5 atmosferas.

Complementariamente a éste, el sistema de láser (17) (18) (19) tipo Smart Clean, de Electronic Engineering, fue empleado en la eliminación de la costra de suciedad de elementos escultóricos y para la limpieza de las áreas en las que no era viable el sistema de limpieza anteriormente mencionado. Los parámetros de ensayo tales como frecuencia, potencia, diámetro del haz y densidad de energía fueron fijados en base a unos ensayos preliminares que aseguraran la limpieza sin dañar el sustrato.

Las grapas metálicas que presentaban procesos de oxidación avanzados, fueron limpiadas, mecánicamente y con disolvente, para la eliminación del óxido con posterior aplicación de un inhibidor de corrosión.

Las cuñas de madera deteriorada fueron sustituidas por rellenos de resinas epoxi, que también se emplearon para rellenar el espacio entre platabanda y piedra.

Las cuñas se empleaban en origen para nivelar y aplomar los sillares, con el tiempo sufren procesos de degradación, generalmente por hongos de pudrición, por lo que es adecuado valorar la necesidad de reconstruir su funcionalidad.

\subsection{Consolidación}

La consolidación efectuada fue de dos tipos: superficial y mecánica.

La primera consistió en la aplicación de éster silícico (silicato de etilo) pulverizado sobre las piedras erosionadas o que habían perdido su cohesión (20). Antes de generalizar la actuación, tal y como se había efectuado con la limpieza, se realizaron diversos ensayos sobre áreas no visibles de la piedra para comprobar el grado de eficacia y compatibilidad de distintos tratamientos. El tratamiento se aplicó en tres capas sucesivas, para asegurar su consolidación superficial.

La consolidación mecánica fue aplicada en aquéllos casos en los que la degradación sufrida era mayor y consistió en el sellado con masilla acrílica, resistente a la radiación UV (13) y la posterior inyección de resinas epoxídicas (consolidantes orgánicos termoendurecibles) de baja viscosidad de los huecos y grietas (13) (21), tal y como puede observarse en la Figura 8.

\subsection{Reintegración de piezas}

Como señalan Esbert et al., ésta es una de las etapas frecuentes en la intervención en elementos pétreos (15). En este caso, dado que la intervención debía de asegurar la durabilidad de las actuaciones, aquellos elementos cuya existencia era necesaria para asegurar la funcionalidad del conjunto fueron recompuestos con morteros (13). Este fue el caso de las cornisas e impostas cuya ausencia generaba escorrentías en fachada y daños por filtración de agua en las piedras. Junto con éstas, algunas dovelas de arcos y sillares cuyas pérdidas volumétricas eran significativas, fueron, igualmente, reintegrados.

Las piezas pétreas fracturadas fueron objeto de dos técnicas. En general, se eligió un sistema de cosido y microcosido con acero inoxidable roscado; no obstante, cuando las piezas presentaban un exceso de fragmentación y dicha técnica no era viable, se optó por una resina de poliéster reforzada con espigas de fibra de vidrio para el anclaje. Los morteros de reintegración fueron en base de cal con una selección cuidada de áridos (16) (22) (23) y dosificados en relación 1:1, en peso. En el caso de los elementos expuestos al agua de la lluvia, a dicha mezcla se añadió una pequeña cantidad de resina acrílica que otorgara mayor impermeabilización. En todos los casos, siguiendo las recomendaciones de otros autores, se evitó que dichos morteros pudieran liberar compuestos solubles que reaccionaran con el soporte (16).

La elección de dicho mortero de reparación (20) estuvo fundamentada en estudios desarrollados in situ para garantizar que las propiedades fueran similares a las del sustrato pétreo y al mismo tiempo mostrara una adherencia adecuada sobre el mismo. La adición de pigmentos permitía, además, que el mortero tuviera una textura y entonación que reintegrara visualmente el conjunto en la distancia. En condiciones de proximidad, la diferencia entre lo original y lo reintegrado se manifestaba a través de la textura superficial (Figura 9 y 10).

\subsection{Tratamiento de juntas}

Los procesos de degradación de las superficies pétreas habían provocado también la pérdida del rejuntado. Estas zonas, junto con las que fueron abiertas al eliminar los morteros de cemento, fueron reintegradas con morteros de cal hidráulica y mezcla de árido calizo y silíceo.

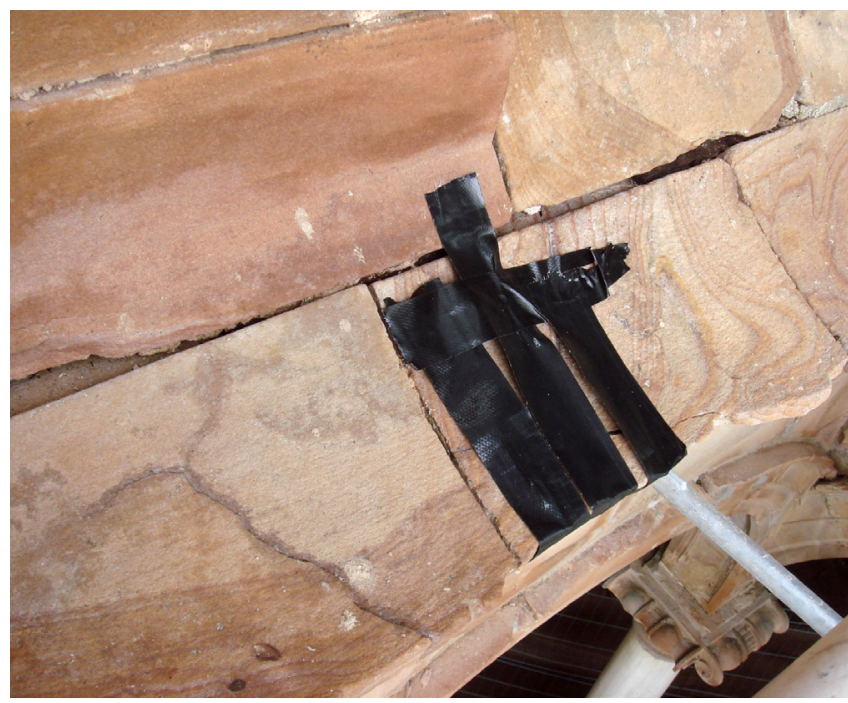

Figura 8. Proceso de inyección de resinas epoxídicas en los huecos y grietas. 


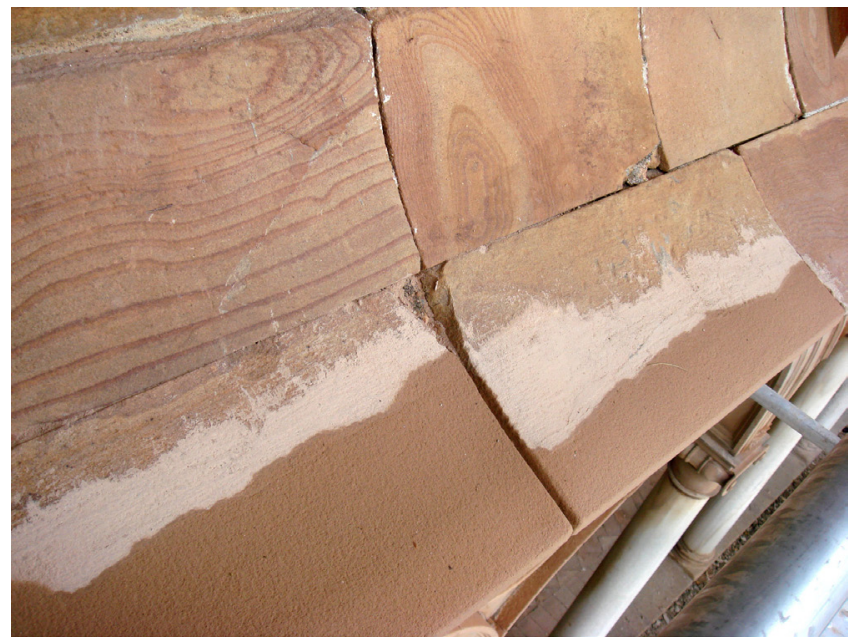

Figura 9. Reintegración de piezas.

\subsection{Reintegración cromática}

Finalmente, los morteros incorporados fueron objeto de estudios cromáticos para su adecuación. Los tonos buscados fueron obtenidos con la incorporación de pigmentos naturales a una solución acuosa de hidróxido cálcico. Dicha solución fue aplicada en sucesivas capas a modo de veladuras.

\section{CONCLUSIONES}

El criterio de intervención así como la metodología a adoptar en cada obra de restauración debe y estará condicionada no sólo por las necesidades de «recuperación» específicas del monumento sino también de las características de la obra.

Asimismo, como ponen de manifiesto las distintas recomendaciones internacionales, la adopción de distintas técnicas y materiales estará asegurada mediante la realización de ensayos y comprobaciones previos in situ en las que se compruebe la adecuación y compatibilidad de las mismas a las condiciones específicas de cada caso.

En el presente artículo se expone el método de intervención seguido en la restauración de las cornisas e impostas del claustro del Convento de la Asunción de Calatravas en Almagro consistente en un primer análisis exhaustivo de la historia, evolución de la edificación y de sus necesidades así

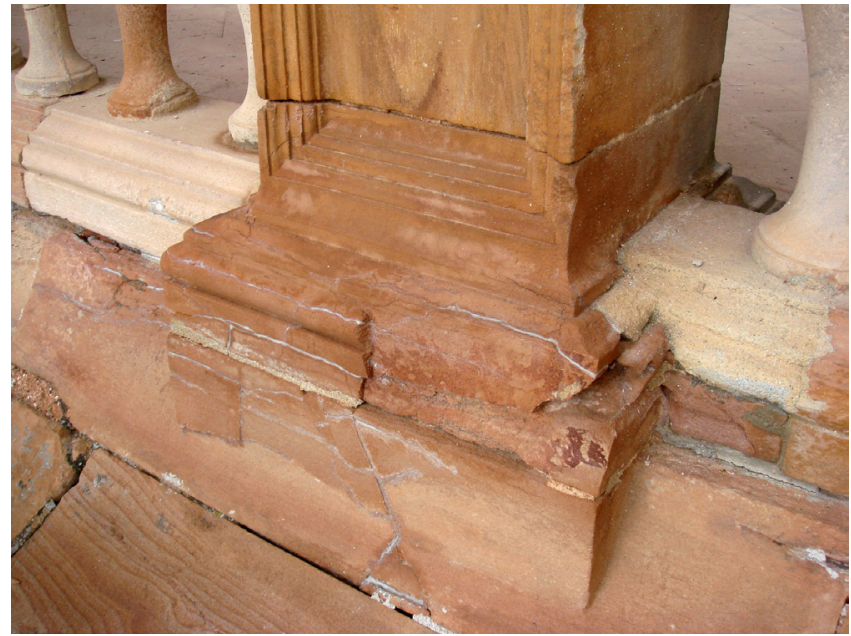

Figura 10. Proceso de recomposición de pie de balaustrada.

como también de los procesos patológicos que le afectan, para posteriormente, abordar la intervención con algunas de las técnicas que comúnmente se emplean en restauración y cuya eficacia ha sido demostrada, no sólo por el paso del tiempo sino también por estudios realizados in situ, teniendo en cuenta que cada caso de intervención es único no sólo por sus características sino también por sus necesidades. En este sentido, se reitera la importancia e interés de la realización de los análisis in situ sobre el propio material a intervenir, lo que asegura no sólo el conocimiento más detallado y preciso del monumento sino también la adecuada elección de las técnicas de restauración disponibles y adaptadas a los condicionantes específicos de cada caso (ubicación y accesibilidad de la zona a intervenir y materiales y estado de conservación, entre otros) al tiempo que se asegura su compatibilidad, previamente a su aplicación sobre una superficie de mayor extensión. Asimismo, la restauración aborda los trabajos que permiten al monumento preservar su aspecto original, recuperando la funcionalidad perdida y garantizando su conservación y mantenimiento a medio y largo plazo.

\section{AGRADECIMIENTOS}

Quisiéramos expresar nuestra gratitud a Vicente Aguilar en cuanto no sólo a las aportaciones al artículo sino también por su experiencia y sugerencias durante el proceso de restauración.

\section{REFERENCIAS}

(1) Díez-de Baldeón, C. (1993). Almagro, arquitectura y sociedad. Toledo: Junta de Comunidades de Castilla-La Mancha.

(2) Díez-de Baldeón, C. (1993). Arquitectura del Renacimiento. En La provincia de Ciudad Real - III. Arte y cultura. Albacete: Diputación de Ciudad Real.

(3) Díez-de Baldeón, C. (1993). Forma, función y símbolo en el Convento de la Asunción de Calatrava. En Historia de Almagro. Ciudad Real: Diputación de Ciudad Real.

(4) Maldonado-Ramos, L., Barbero-Barrera, M.M., Cercós-García, L.F., Rivera-Gámez, D., Urquiza-Bustinza, D., Vela-Cossío, F. (2002). Plan Director de Restauración del Convento de la Asunción de Calatravas (Almagro, Ciudad Real). (No publicado).

(5) Maldonado-Ramos, L., Rivera-Gámez, D., Vela-Cossío, F. (Eds.). (2005). Los estudios preliminares en la restauración del patrimonio arquitectónico. Madrid: Mairea.

(6) González Moreno-Navarro, A. (1996). La restauración de la Iglesia de Santa María del Castillo de Castelldefels (Barcelona, España). Informes de la Construcción, 48(445): 33-49, doi: http://dx.doi.org/10.3989/ic.1996.v48.i445.991. 
(7) Alonso, F.J., Díaz-Pache, F., Esbert, R.M. (1994). Interacción piedra-ambiente, $1^{0}$ parte: Formas de alteración desarrolladas sobre la piedra de la Catedral de Burgos. Informes de la Construcción, 46(433): 5-18, doi: http://dx.doi. org/10.3989/ic.1994.v46.i433.1113.

(8) García-de Miguel, J.M. (1999). Procesos de degradación de la piedra. En Mas-Guindal, L. (Ed.). Procedimientos y técnicas constructivas del Patrimonio (pp. 175-183). Madrid: Munilla-Lería.

(9) AEMET. (2013). Datos climáticos normales. www.aemet.es.

(10) Ruedrich, J., Kirchner, D., Siegesmund, S. (2011). Physical weathering of building stones induced by freeze-thaw action: a laboratory long-term study. Environmental Earth Science, 63(7-8): 1573-1586, doi: http://dx.doi.org/10.1007/ s12665-010-0826-6.

(11) Grossi, C.M., Esbert, R.M., Díaz-Pache, F. (1998). Degradación y durabilidad de materiales rocosos de edificación en ambientes urbanos. Materiales de Construcción, 48(252): 5-25, doi: http://dx.doi.org/10.3989/mc.1998.v48.i252.461.

(12) Rodríguez-Navarro, C., Sebastian, E. (1996). Role of particulate matter from vehicle exhaust on porous building stones (limestone) sulfation. The Science of the Total Environment 187(2): 79-91, doi: http://dx.doi.org/10.1016/o0489697(96)05124-8.

(13) García-de Miguel, J.M. (2009). Tratamiento y conservación de la piedra, el ladrillo y los morteros en monumentos y construcciones. Madrid: Consejo General de la Arquitectura Técnica de España.

(14) Garabito, J., Manso, J.M., Rodríguez, A., Garabito, J.C., Calderón, V. (2013). Métodos para la limpieza física, química y biológica de las fábricas de piedra de monumentos históricos. El caso de San Lorenzo del Real de Burgos. Informes de la Construcción, 65(531): 367-380, doi: http://dx.doi.org/10.3989/ic.12.018.

(15) Esbert, R.M., Ordaz, J., Alonso, F.J., Montoto, M. (1997). Manual de diagnosis 5. Manual de diagnosis y tratamiento de materiales pétreos y cerámicos. Barcelona: Colegio de Aparejadores y Arquitectos Técnicos de Barcelona.

(16) Luxán, M.P., Dorrego, F. (2003). Caracterización y selección de morteros en la restauración de la Portada de los Reyes (Benavente, Zamora). Materiales de Construcción, 54(273): 35-44, doi: http://dx.doi.org/10.3989/mc.2004.v54. i273.221.

(17) Bromblet, P., Labouré, M., Orial, G. (2003). Diversity of the cleaning procedures including laser for the restoration of carved portals in France over the last 10 years. Journal of Cultural Heritage, 4: 17-26, doi: http://dx.doi.org/10.1016/ S1296-2074(02)01222-0.

(18) Sabatini, G., Giamello, M., Pini, R., Siano, S., Salimbeni, R. (2000). Laser cleaning methodologies for stone façades and monuments: laboratory analyses on lithotypes of Siena architecture. Journal of Cultural Heritage, 1: 9-19, doi: http:// dx.doi.org/10.1016/S1296-2074(00)oo144-8.

(19) Labouré, M., Bromblet, P., Orial, G., Wiedemann, G., Simon-Boisson, C. (2000). Assessment of laser cleaning rate on limestones and sandstones. Journal of Cultural Heritage, 1: 21-27, doi: http://dx.doi.org/10.1016/S1296-2074(00)00195-3.

(20) García-de Miguel, J.M. (1999). Metodología del diagnóstico y tratamiento. En Mas-Guindal Lafarga (Ed.). Procedimientos y técnicas constructivas del Patrimonio (pp. 185-213). Madrid: Munilla-Lería.

(21) Carbonell-de Masy, M. (1933). Conservación y Restauración de Monumentos: piedra, cal, arcilla. Barcelona.

(22) Moropoulou A., Bakolas, A., Bisbikou, K. (2000). Investigation of the technology of historic mortars. Journal of Cultural Heritage 1(1): 45-48, doi: http://dx.doi.org/10.1016/S1296-2074(99)00118-1.

(23) Faria, P., Henriques, F., Rato, V. (2008). Comparative evaluation of lime mortars for architectural conservation. Journal of Cultural Heritage, 9(3): 338-346, doi: http://dx.doi.org/10.1016/j.culher.2008.03.003. 